\title{
Embedding Medium
}

National Cancer Institute

\section{Source}

National Cancer Institute. Embedding Medium. NCI Thesaurus. Code C113307.

A material that infiltrates and supports a specimen and preserves its shape and structure for sectioning and microscopy. 